\title{
The Psychological Impact of Epidemic and Pandemic Outbreaks on Healthcare Workers: Rapid Review of the Evidence
}

\author{
Emanuele Preti ${ }^{1,2} \cdot$ Valentina Di Mattei ${ }^{3,4} \cdot$ Gaia Perego $^{1} \cdot$ Federica Ferrari $^{4} \cdot$ Martina Mazzetti $^{4} \cdot$ Paola Taranto $^{4}$. \\ Rossella Di Pierro ${ }^{1,2} \cdot$ Fabio Madeddu ${ }^{1,2} \cdot$ Raffaella Calati ${ }^{1,5}$
}

Published online: 10 July 2020

(C) Springer Science+Business Media, LLC, part of Springer Nature 2020

\begin{abstract}
Purpose of Review We aim to provide quantitative evidence on the psychological impact of epidemic/pandemic outbreaks (i.e., SARS, MERS, COVID-19, ebola, and influenza A) on healthcare workers (HCWs).

Recent Findings Forty-four studies are included in this review. Between 11 and $73.4 \%$ of HCWs, mainly including physicians, nurses, and auxiliary staff, reported post-traumatic stress symptoms during outbreaks, with symptoms lasting after 1-3 years in $10-40 \%$. Depressive symptoms are reported in $27.5-50.7 \%$, insomnia symptoms in 34-36.1\%, and severe anxiety symptoms in $45 \%$. General psychiatric symptoms during outbreaks have a range comprised between 17.3 and $75.3 \%$; high levels of stress related to working are reported in 18.1 to $80.1 \%$. Several individual and work-related features can be considered risk or protective factors, such as personality characteristics, the level of exposure to affected patients, and organizational support.

Summary Empirical evidence underlines the need to address the detrimental effects of epidemic/pandemic outbreaks on HCWs' mental health. Recommendations should include the assessment and promotion of coping strategies and resilience, special attention to frontline HCWs, provision of adequate protective supplies, and organization of online support services.
\end{abstract}

Keywords Healthcare workers · Mental health $\cdot$ Psychological distress $\cdot$ Pandemic $\cdot$ Epidemic $\cdot$ COVID-19

This article is part of the Topical Collection on Personality Disorders

Electronic supplementary material The online version of this article (https://doi.org/10.1007/s11920-020-01166-z) contains supplementary material, which is available to authorized users.

Emanuele Preti

emanuele.preti@unimib.it

1 Department of Psychology, University of Milan-Bicocca, Piazza dell'Ateneo Nuovo, 1, 20126 Milan, Italy

2 Centro per lo studio e la terapia dei disturbi della personalità (C.R.E.S.T.), Milan, Italy

3 School of Psychology, Vita-Salute San Raffaele University, Via Olgettina, 58, 20132 Milan, Italy

4 Clinical and Health Psychology Unit, IRCCS San Raffaele Scientific Institute, Via Olgettina, 60, 20132 Milan, Italy

5 Department of Adult Psychiatry, Nîmes University Hospital, 4 Rue du Professeur Robert Debré, 30029 Nimes, France

\section{Introduction}

In December 2019, an outbreak of a novel coronavirus pneumonia, namely, coronavirus disease 19 (COVID-19), hit Wuhan (Hubei, China). During the following weeks, other significant outbreaks of COVID-19 were reported across the world and the World Health Organization (WHO) declared the COVID-19 outbreak a global pandemic on 11 March 2020 .

In the last 20 years, other outbreaks of novel infectious diseases occurred all over the world. Recent examples are the outbreak of severe acute respiratory syndrome (SARS) in 2002 and the 2009-2010 A/H1N1 influenza pandemic. Overall, pandemic situations require intense and immediate response in terms of healthcare, with thousands of healthcare workers (HCWs), either directly (e.g., physicians, nurses) or indirectly (e.g., aides, laboratory technicians, and medical waste handlers) delivering care to patients, fighting at the frontline to address the challenges posed to healthcare systems by the almost three million patients infected by the time we are writing. 
HCWs are thus facing critical situations that increase their risk of suffering for the psychological impact of dealing with a number of unfavorable conditions, with consequences that might span from psychological distress to mental health symptoms.

\section{Why Is This Review Needed?}

HCWs responding to a pandemic outbreak are exposed to physical and psychological stressors that may result in severe mental health outcomes. Furthermore, healthcare workforces play a crucial role in successfully responding to a pandemic situation. In this sense, potential psychological negative consequences not only are detrimental to HCWs' well-being but might also reduce their ability to address effectively the heath emergency.

The worldwide spread of COVID-19 is challenging the capacity of response of healthcare systems, and policymakers need evidence to address the issue of psychological distress and mental health of HCWs, given their role in responding to the situation. WHO recommends rapid reviews of empirical evidence in these circumstances [1], in order to give recommendations that may help strengthening the response capacity of healthcare systems.

For this reason, we performed a review of empirical studies on the impact of epidemic and pandemic outbreaks on healthcare providers in terms of psychological distress and mental health. Our review is aimed at providing evidence on maladaptive psychological outcomes in HCWs facing epidemic/pandemic situations. Moreover, we aim to identify potential risk and protective factors for such maladaptive consequences.

\section{Methods}

\section{Search Strategy and Selection Criteria}

We systematically searched potentially eligible articles on PubMed, PsycINFO, and Web of Science databases on 30 March 2020. We used the following combinations of terms: "infect*”, "COVID*”, "SARS”, "influenza", "flu”, "MERS”, "ebola", "Mental”, "Psych*”, "Health Personnel”, "health worker", "Medical Staff", "Physician", and "Nurses". The full search strategy is available in Appendix 1. We developed the following set of inclusion criteria for papers to be included in our review:

1. Studies had to report on primary research

2. Studies had to be published in a peer-reviewed journal

3. Studies had to be written in English

4. Studies had to include data on healthcare providers' mental health or psychological well-being or data on factors associated with healthcare providers' mental health or psychological well-being during epidemic/pandemic (i.e., SARS, Middle East respiratory syndrome (MERSCoV), COVID-19, ebola virus disease (EVD), and influenza $\mathrm{A}(\mathrm{A} / \mathrm{H} 1 \mathrm{~N} 1$ and $\mathrm{A} / \mathrm{H} 7 \mathrm{~N} 9))$

Excluding criteria were as follows:

1. Qualitative studies

2. Studies focused on distress prevention programs during epidemic/pandemic

3. Studies focused on emergency situations not related to epidemic/pandemic (i.e., wars, natural disasters, and terroristic attacks)

We removed duplicates through Zotero software version 5.0. In the first stage, four independent researchers (GP, PT, MM, and FF) screened titles and abstracts of the papers we found. In the second stage, the same researchers screened the full texts of all remaining studies to assess their eligibility. In both stages, disagreements were solved through discussion with EP and RC.

\section{Data Extraction}

The authors extracted data about the following: publication year, country of study, the type of epidemic/pandemic, participant information (number, occupation), design, assessment scales, time period of study, and main results on psychological outcomes.

To synthesize the data, we performed a qualitative synthesis of findings. We first described psychiatric and psychological difficulties of HCWs during and after epidemics/pandemics; then, we summarized risk and protective factors related to these psychological outcomes.

\section{Results}

\section{Study Selection and Characteristics}

Database search identified 5167 articles; after duplicate removal, 4203 potentially eligible studies remained. After reading titles and abstracts, we excluded 4124 studies; of the remaining 79 studies, 44 met inclusion criteria and were thus included for qualitative synthesis after full-text reading (see Fig. 1 for study selection and Table 1 for a summary of the included studies).

Among the included studies, $27(62 \%)$ referred to the SARS outbreak, $5(11 \%)$ to the MERS-CoV outbreak, 5 $(11 \%)$ to the COVID-19 outbreak, $3(7 \%)$ to the A/H1N1 influenza outbreak, $3(7 \%)$ to the EVD outbreak, and $1(2 \%)$ to the A/H7N9 influenza outbreak. 
Fig. 1 Study selection

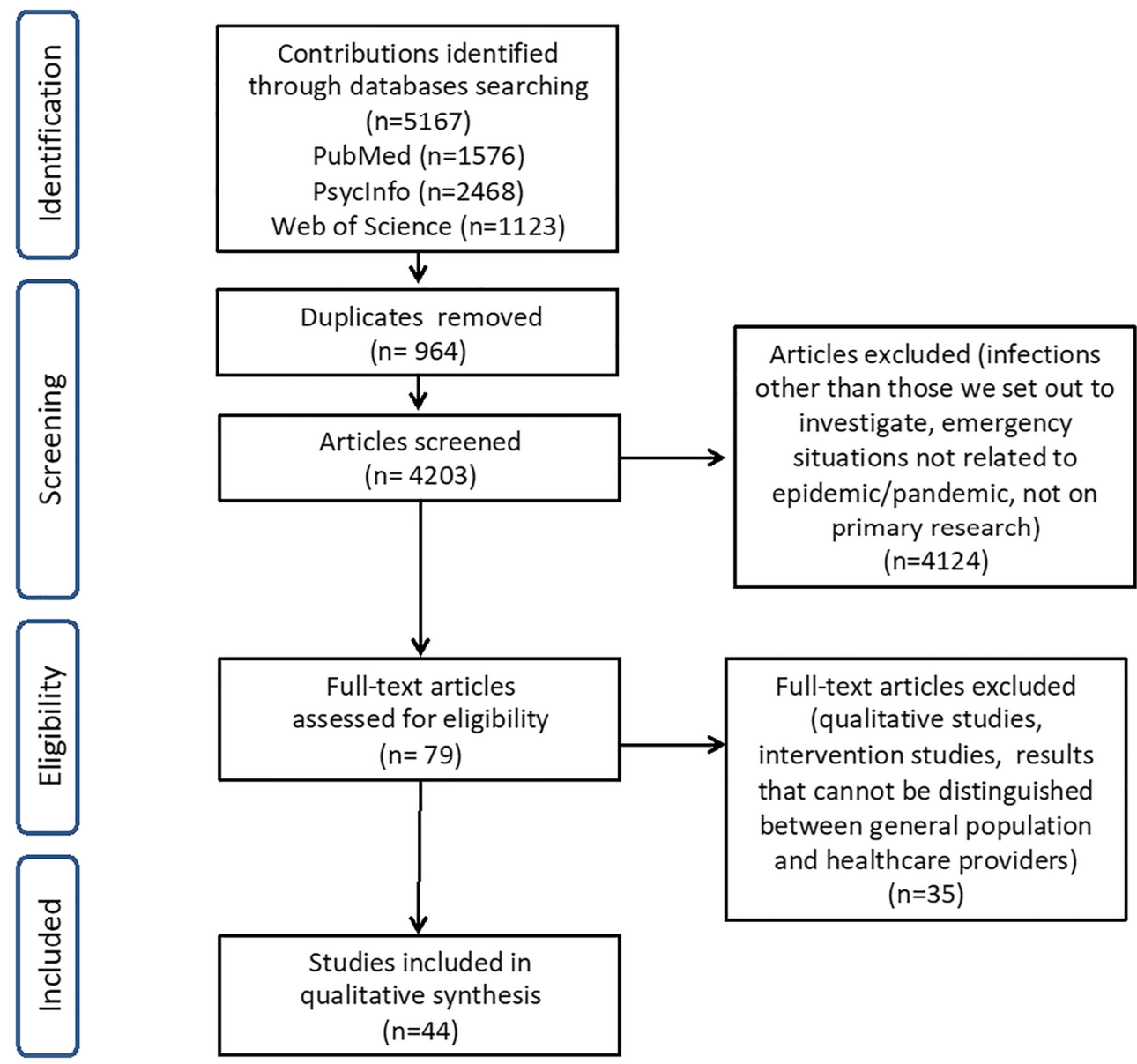

The studies were conducted in different countries: China (19 studies), Canada (eight studies), Taiwan (seven studies), South Korea (two studies), Saudi Arabia (two studies), Greece (one study), Nigeria (one study), Sierra Leone (one study), Liberia (one study), Singapore (one study), and Japan (one study).

Thirty-four studies (77\%) considered only HCWs, namely, physicians, nurses, and auxiliaries, whereas both HCWs and other staff members such as administrative workers and technicians were included in ten studies $(23 \%)$.

All the studies investigated psychological outcomes by means of both validated questionnaires and interviews and/ or study-specific measures.

\section{Psychopathological Symptoms}

\section{Post-traumatic Stress Symptoms}

Post-traumatic stress reactions were examined in 23 studies [3, 6, 8, 13, 15••, 16-18, 22-24, 28-30, 31••, 35-38, 40, 41, 44]. See Table 2 for a detailed description of psychological outcomes included in each study and their findings.

During outbreaks, the prevalence of PTSD-like symptoms was comprised between 11 and $73.4 \%$. Moreover, $51.5 \%$ of
HCWs scored above the Impact of Event Scale-Revised (IESR) threshold for a PTSD diagnosis [6, 15••, 18, 24]. Studies on the COVID-19 pandemic [15••, 24] reported the highest prevalence rate $(71.5-73 \%)$. In contrast, only $5 \%$ of the staff members of a psychiatric hospital met the DSM-IV criteria for an acute stress disorder during the SARS outbreak [3]. However, the authors underlined that the specific type of institution they considered limits the generalizability of this result [3].

Other studies examined PTSD manifestations after the end of the outbreaks. Findings suggest that from 18.6 to $28.4 \%$ of HCWs still have significant PTSD symptoms after 1 month from the end of the pandemic [22, 36, 38], 17.7\% after 6 months [35, 41], and 10-40\% after $1-3$ years [30, 40, 44].

Two case-control studies observed that HCWs showed a significant higher post-event morbidity to outbreak exposure, compared with non-HCWs $[17,36]$.

\section{Depression and Anxiety Symptoms}

Depressive symptoms were examined in seven studies [15••, $17,21,23,24,31 \bullet \bullet, 38]$. During the acute phase of pandemic, the prevalence of depressive symptomatology was between 27.5 and $50.7 \%$ in HCWs [15••, 24, 38], with higher rates during the COVID-19 pandemic (50.4-50.7\%), compared 


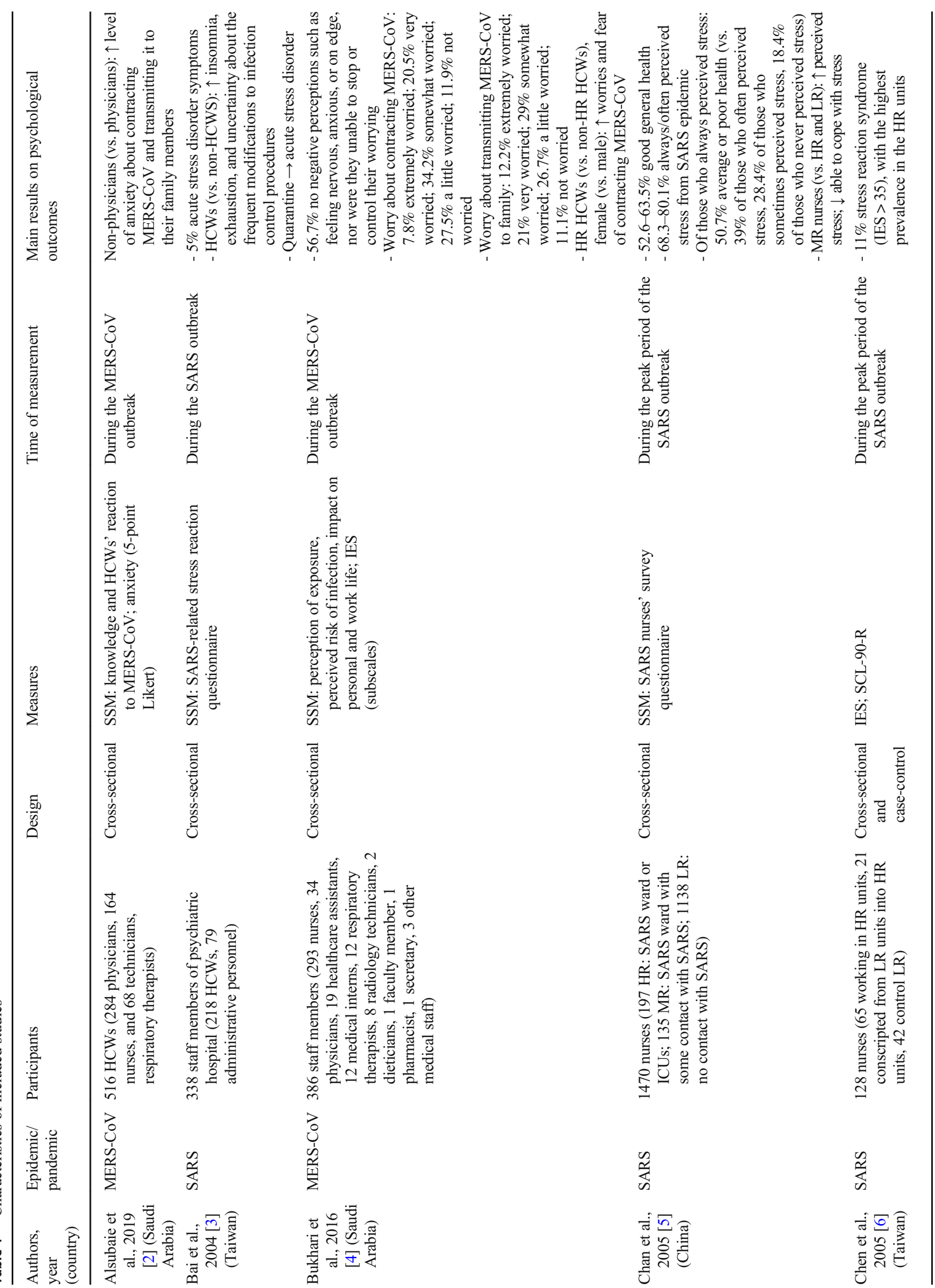




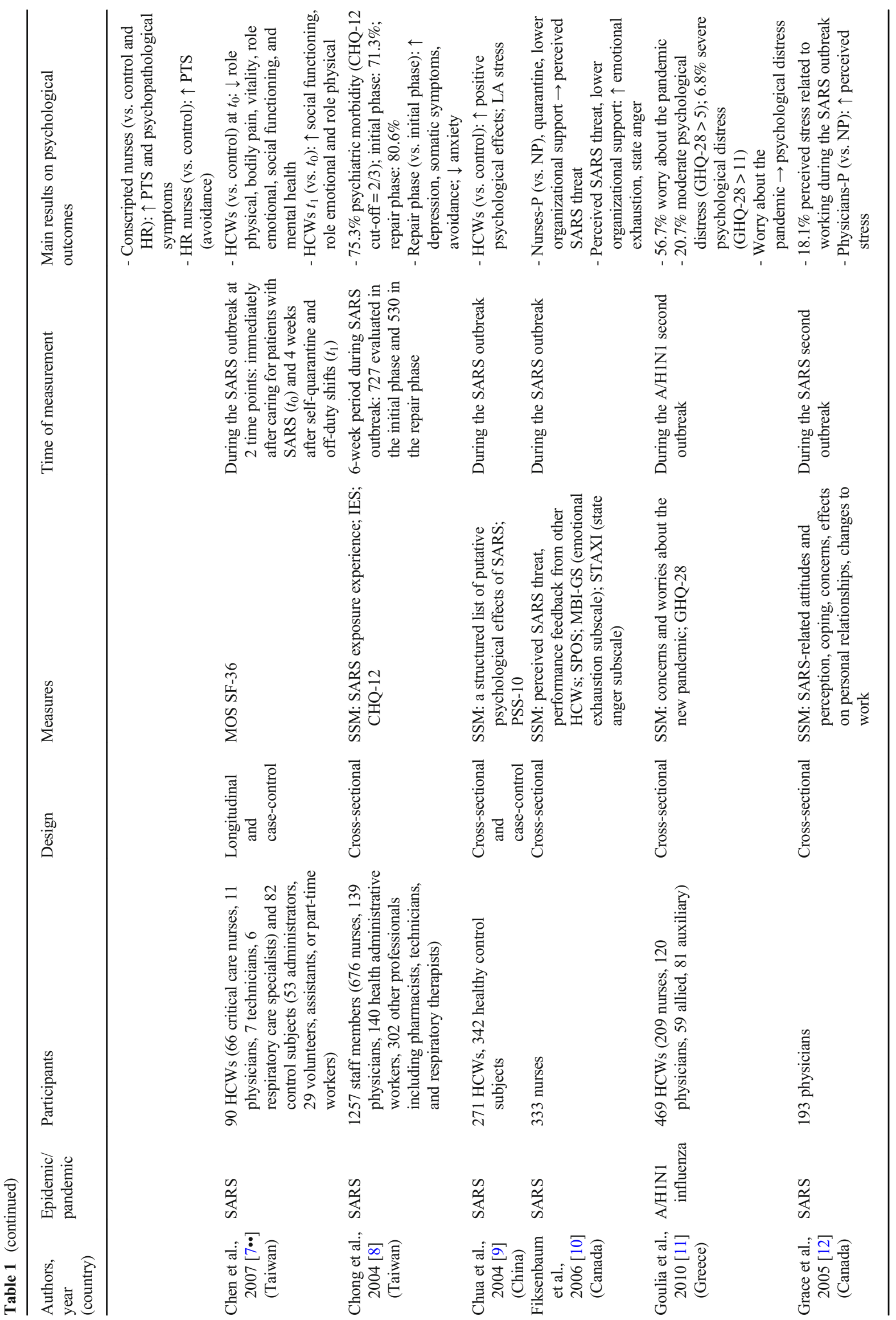




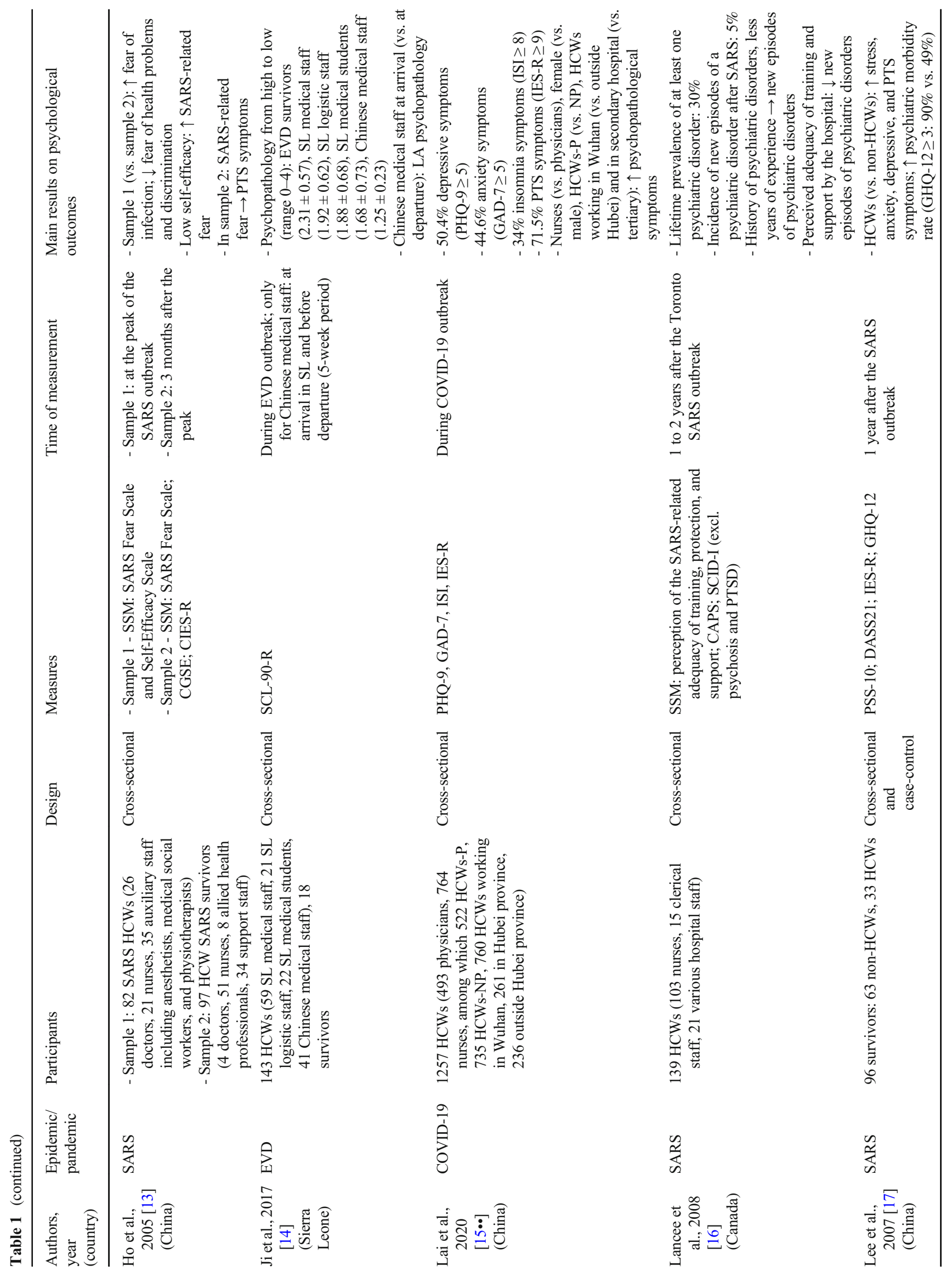




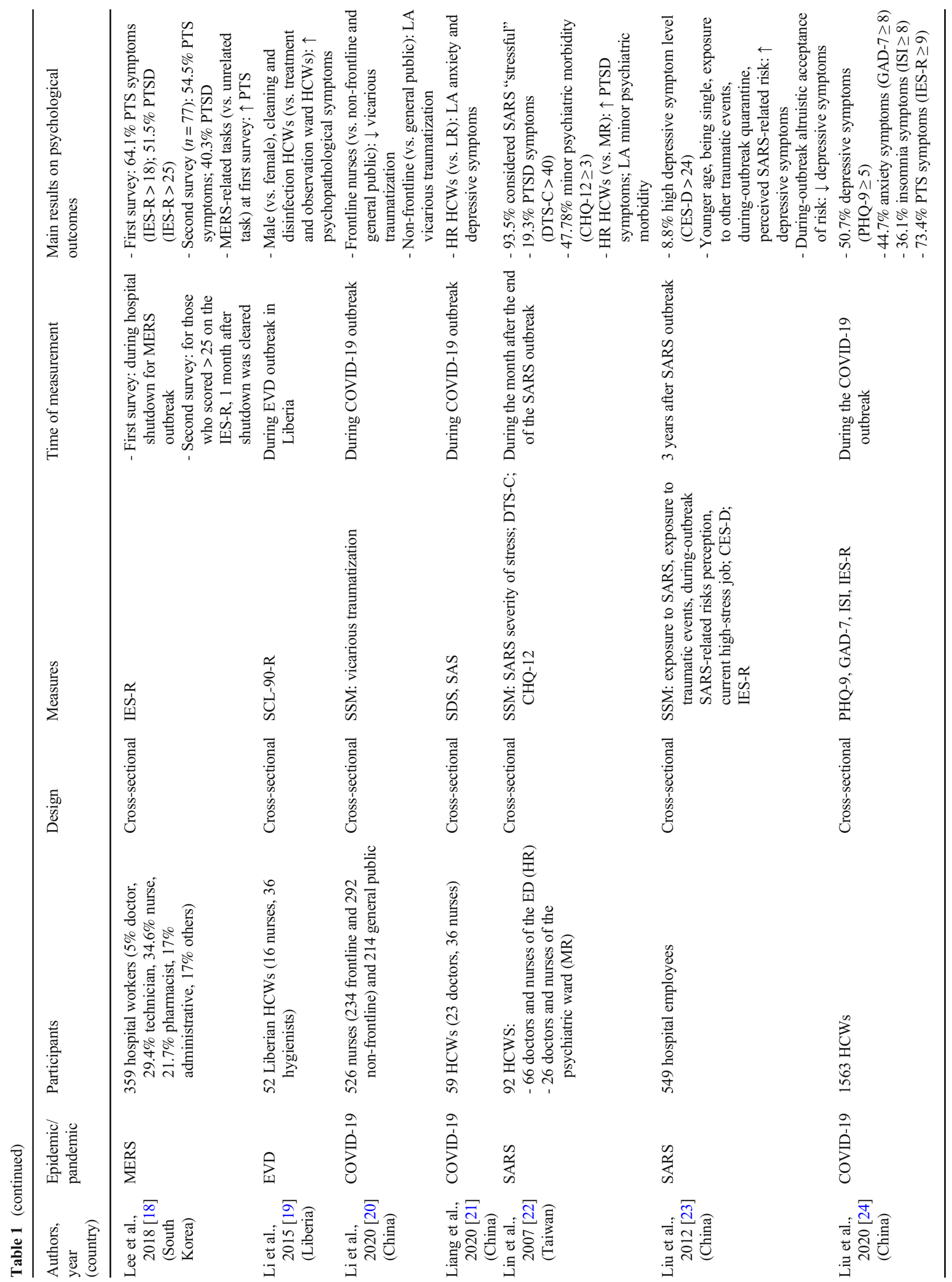




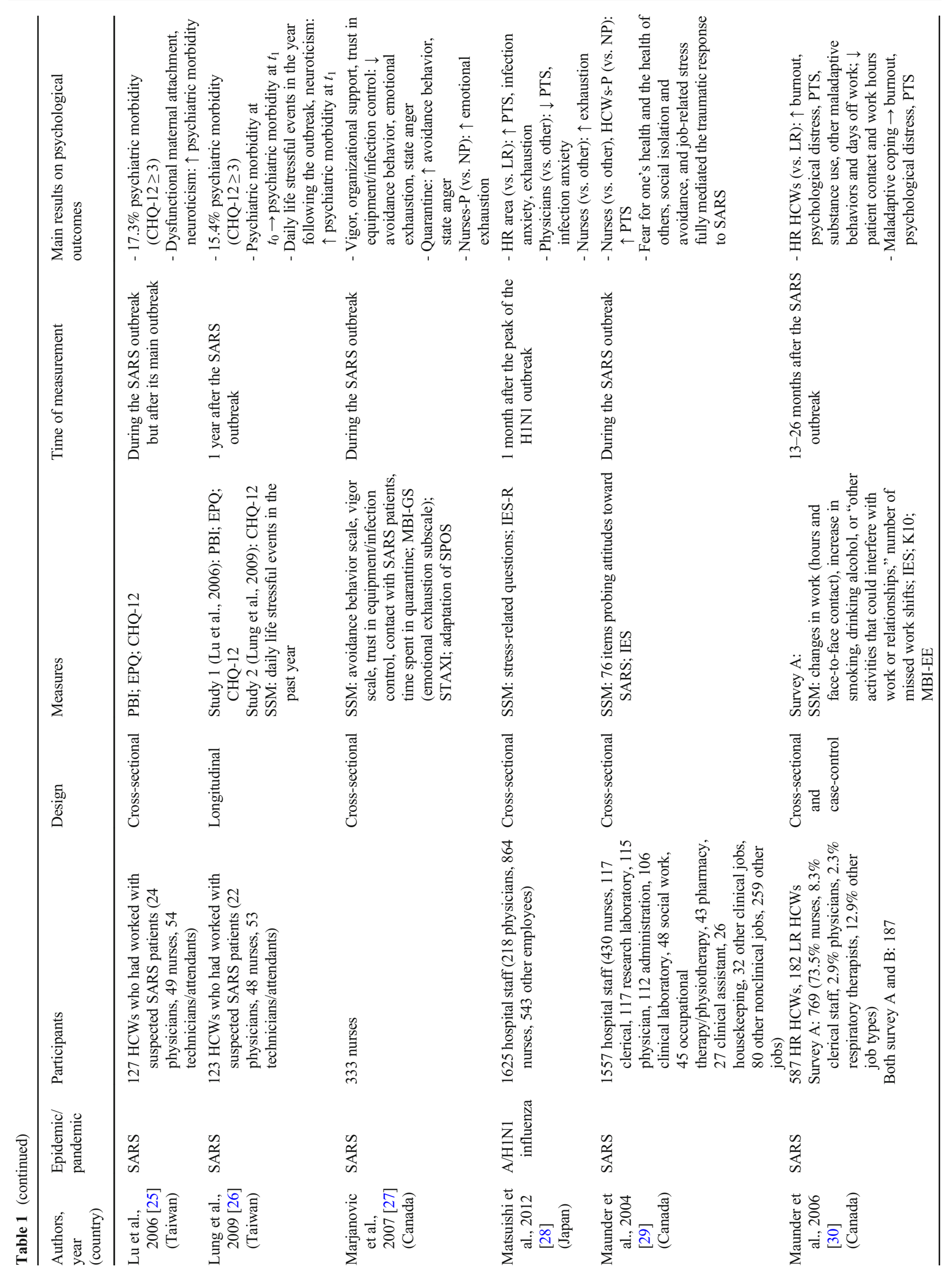




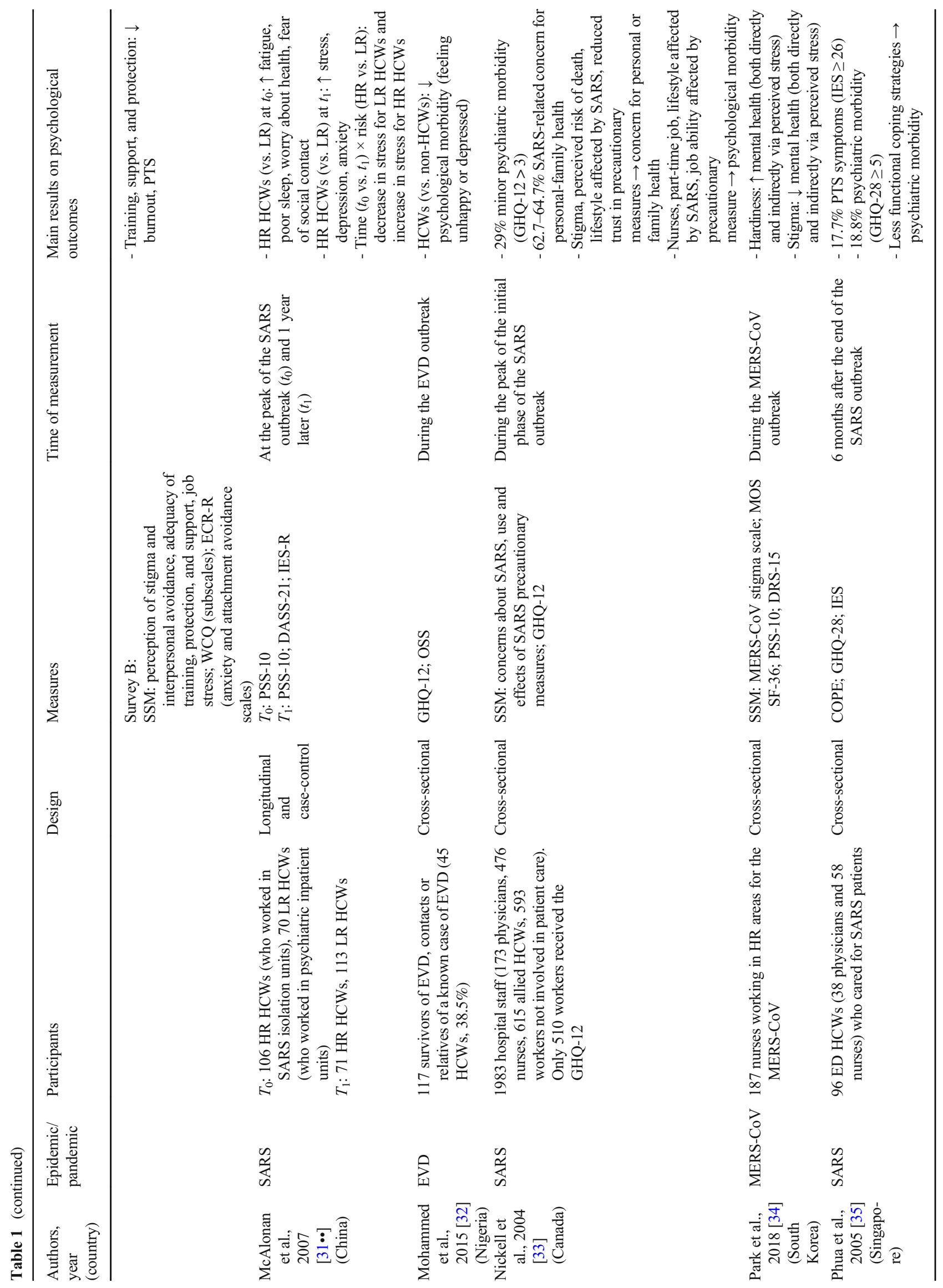




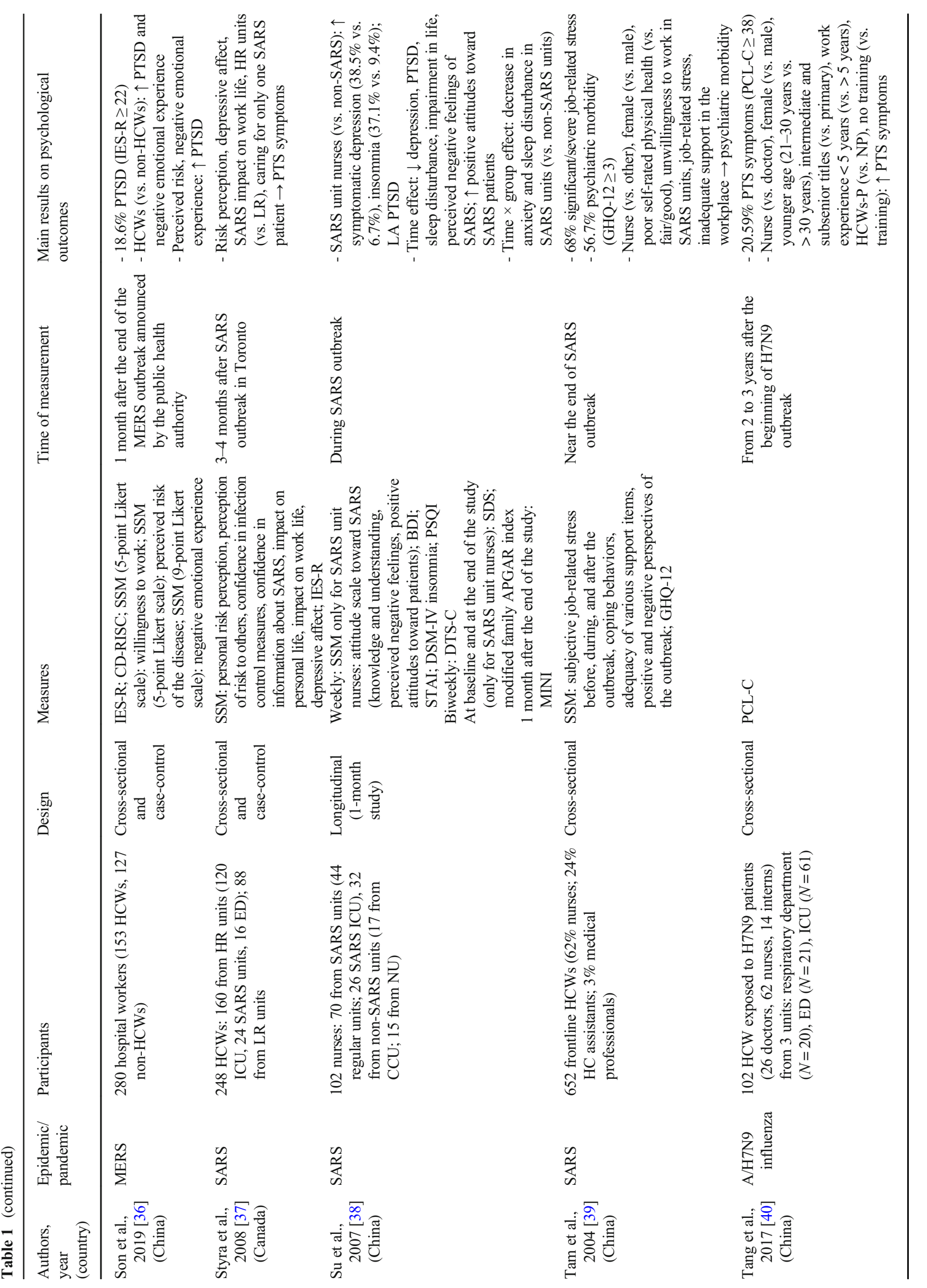




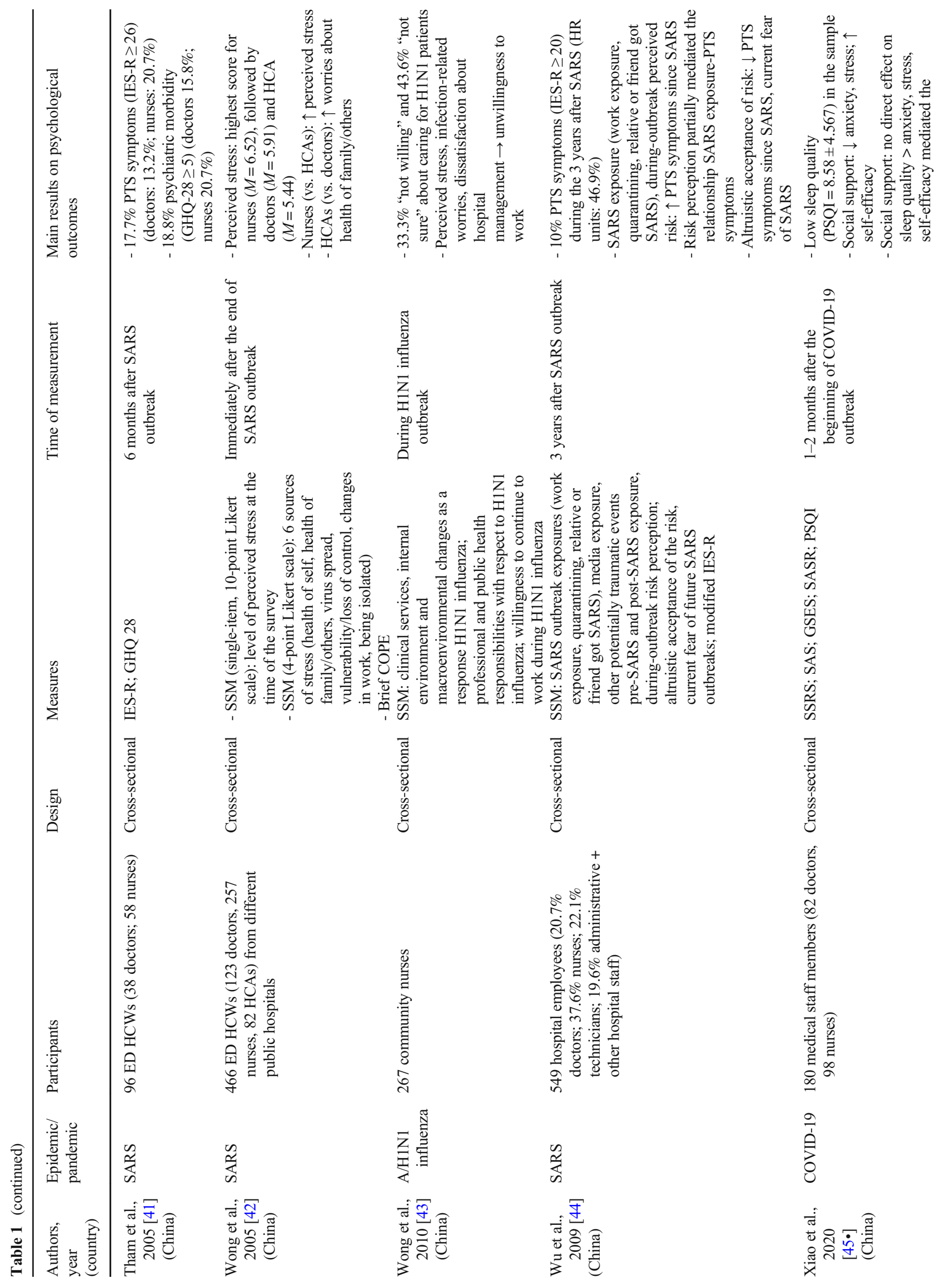




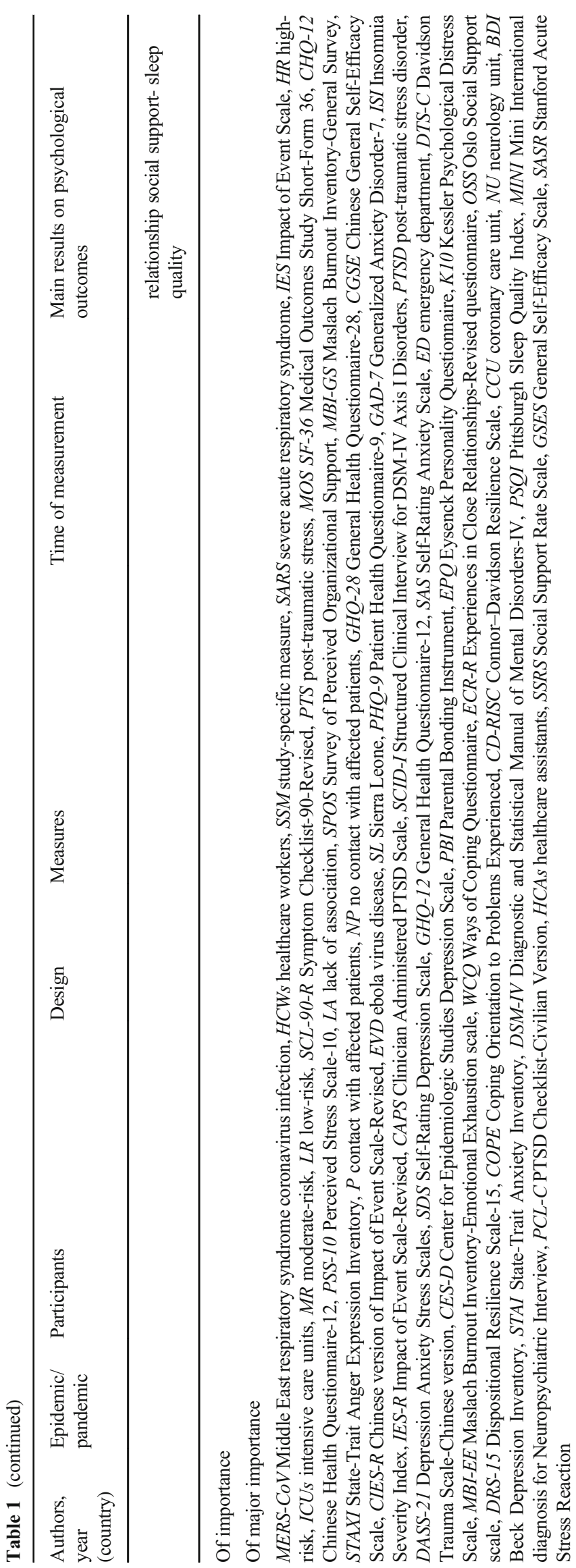




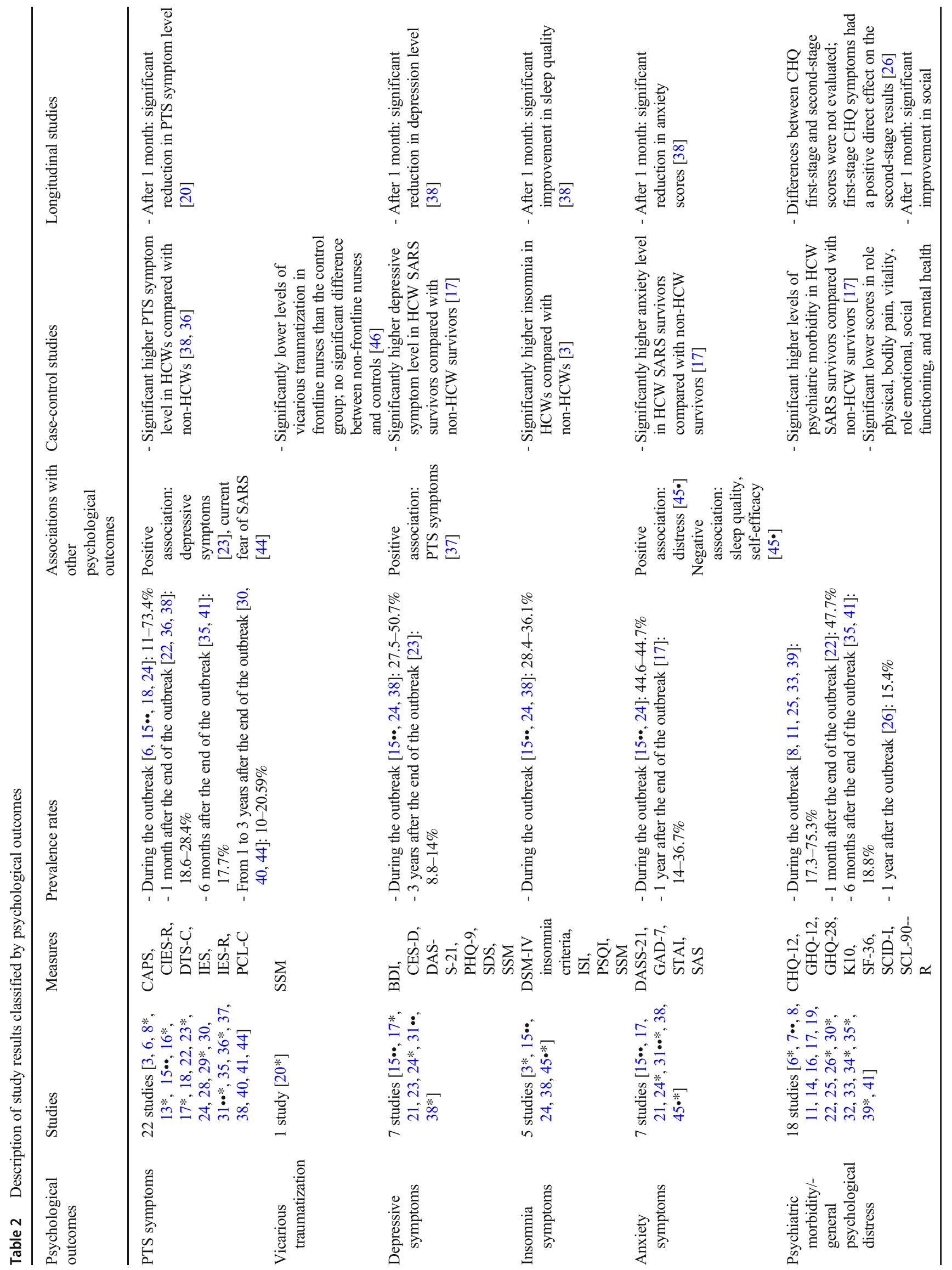




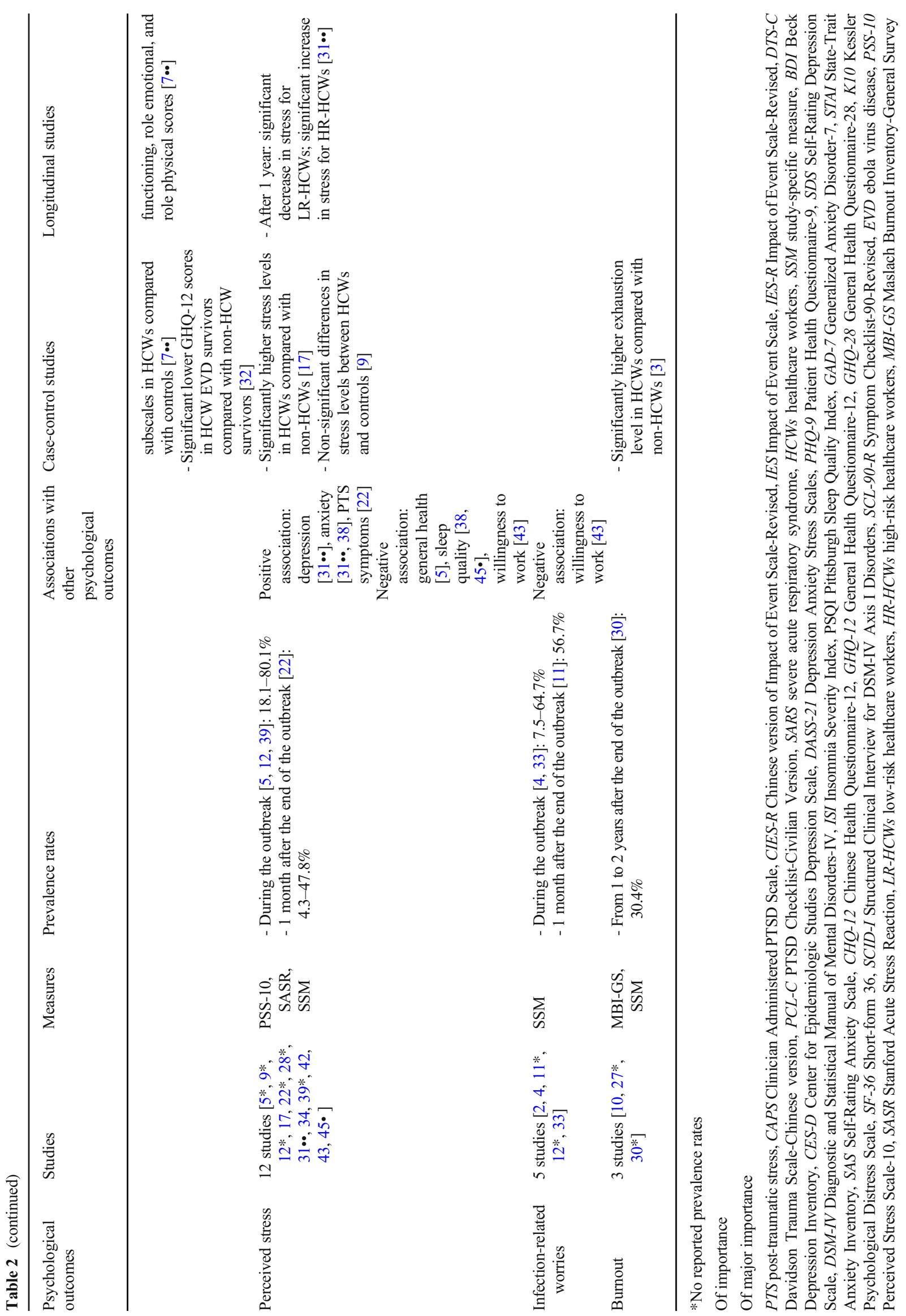


with SARS outbreak (27.6\%). Three years after the end of the Beijing SARS epidemic, about $14 \%$ of the interviewed hospital staff members still showed moderate depressive symptoms, and $8.8 \%$ reported high symptom levels [23].

Five studies specifically investigated insomnia and sleep quality $[3,15 \bullet \cdot, 24,38,45 \bullet$. The DSM-IV criteria for insomnia were met in $28.4 \%$ of nurses, with the highest insomnia rate for the regular SARS unit (50\%), followed by the SARS intensive care unit (23\%), whereas no cases were found in non-SARS units [38]. Significant self-reported insomnia symptoms were observed in $34-36.1 \%$ of COVID-19 HCWs [15••, 24]. Moreover, low sleep quality was reported by medical staff members treating COVID-19 patients [45•].

Five studies investigated anxiety symptoms through standardized self-reported questionnaires during outbreaks $[15 \bullet \bullet$, $21,24,38,45 \cdot$, and two studies examined anxiety symptoms 1 year after pandemic resolution [17, 31••]. Two extensive studies conducted during the peak phase of COVID-19 pandemic reported that about $45 \%$ of HCWs presented severe anxiety symptoms $[15 \bullet \cdot, 24]$. Among medical staff members treating COVID-19 patients, anxiety levels affected psychological well-being, by increasing levels of distress and decreasing sleep quality and self-efficacy [45•].

One study prospectively evaluated changes in anxiety symptoms in a sample of SARS nurses over time [38]. Findings suggest a significant reduction in both depressive and anxiety symptoms at 1-month follow-up, as well as significant improvement in sleep quality.

In a case-control study, Lee and colleagues [17] found significantly higher depressive and anxiety symptom levels among HCW survivors compared with non-HCW survivors 1 year after the end of the SARS epidemic.

\section{Psychiatric Morbidity and General Psychological Distress}

Eighteen studies investigated HCWs' mental health using screening measures of psychiatric symptoms and psychological distress and questionnaires of general health status [6-8, $11,14,16,17,19,22,25,26,30,32-35,39,41]$.

Specifically, five studies investigated psychiatric symptoms during outbreaks through the General Health Questionnaire, reporting a considerable variability in prevalence rates, with a range comprised between 17.3 and $75.3 \%$ $[8,11,25,33,39]$.

One month after the end of the SARS outbreak, $47.8 \%$ of hospital staff members showed psychiatric symptoms [22]. Six months after the SARS epidemic, $18.8 \%$ of HCWs presented psychiatric symptoms [35, 41].

Between 1 and 2 years after SARS resolution, the incidence of new episodes of psychiatric disorders (diagnosed with the SCID-I, excluding the psychotic disorders and PTSD) was of $5 \%$ in HCWs in Toronto [16]. Similarly, 1 year after the end of the SARS epidemic, $15.4 \%$ of the respondents still showed significant mental health symptoms [26].

One year after the end of the SARS outbreak, HCW survivors had a sixfold increased risk for psychiatric symptoms, compared with non-HCW survivors, even after controlling for age, sex, and education level [17]. Conversely, in a study on EVD survivors, Mohammed and colleagues [32] found that being a $\mathrm{HCW}$ was a protective factor for depressive feelings.

\section{Psychological Impact}

\section{Perceived Stress}

Twelve studies investigated HCWs' perceived stress during outbreaks $[5,9,12,17,22,28,31 \bullet \bullet, 34,39,42,43,45 \bullet]$. From 18.1 to $80.1 \%$ of HCWs reported high levels of work-related stress during the SARS outbreak [5, 12, 39].

Moreover, HCW survivors reported higher perceived stress than survivors from the general population 1 year after the end of the SARS outbreak [17]. Conversely, Chua and colleagues [9] found no significant differences in perceived stress between HCWs and healthy control subjects after the SARS outbreak.

One prospective study [31・•] examined changes in perceived stress among high-risk and low-risk HCWs over time, from the peak of the SARS outbreak to 1 year after resolution. The authors observed a significant time $\times$ risk interaction effect, with a general trend toward a decrease of perceived stress for low-risk HCWs and an increase of perceived stress for high-risk HCWs.

\section{Infection-Related Worries}

Five studies specifically investigated HCWs' infection-related worries through non-standardized ad hoc measures $[2,4,11$, 12, 33].

Nickell and colleagues [33], in a study on a large sample of hospital staff members, found that $64.7 \%$ of the respondents expressed SARS-related concerns for personal health and $62.7 \%$ for family health during the peak phase of the outbreak. Bukhari and colleagues [4] examined fears related to contracting and transmitting MERS-CoV in a sample of Saudi Arabian HCWs working during the outbreak. The authors found that from 7.8 to $20.5 \%$ of the respondents were extremely very worried about contracting MERS-CoV over the past 4 weeks; from 12.2 to $21 \%$ of the sample reported to be extremely very worried about transmitting the infection to family members or friends. One month after the end of the A/ H1N1 pandemic, Goulia and colleagues [11] found that $56.7 \%$ of their sample still expressed a moderately high level of concern about the disease. 


\section{Burnout}

Three studies investigated HCWs' burnout reactions to outbreaks, specifically examining its emotional exhaustion component $[10,27,30]$. One to 2 years after the SARS outbreak, $30.4 \%$ of HCWs who had direct contact with infected patients still reported a high level of emotional exhaustion [30].

Bai and colleagues [3] found a significantly higher level of emotional exhaustion among SARS HCWs compared with non-HCWs. However, the authors measured emotional exhaustion through a single item.

\section{Risk and Protective Factors}

We report detailed results regarding risk and protective factors for the aforementioned mental health outcomes in Table 3 . As shown, sociodemographic factors, such as gender, age, education, marital status, and having children, as well as their association with mental health outcomes, were extensively investigated. However, results are mixed (see Table 3).

Some studies investigated the role of personal variables, such as personality, attachment styles, coping strategies, and clinical features, on mental health outcomes. Specifically, neuroticism [25, 26], dysfunctional attachment [25, 30], and maladaptive coping $[30,35]$ were found to be risk factors for mental health outcomes. Additionally, resilience indicators (i.e., hardiness, vigor) [27, 34] and self-efficacy [13, 45•] were protective factors for mental health outcomes.

Few retrospective studies found that having a past psychiatric history $[16,38]$ and reporting traumatic and stressful life events $[23,26,44]$ were risk factors for psychiatric disorders or symptoms, respectively.

Several work-related features were investigated as factors associated with mental health outcomes: occupation, years of professional experience, working in high-risk units, direct contact with affected patients, being quarantined, being infected, or having relatives/friends get infected, confidence in equipment and protective measures, perceived organizational support, perceived adequacy of training, disease-related risk perception, job-related stress, and confidence in disease-related information.

Four studies found that physicians were less worried and anxious about the infection, compared with other HCWs [2, 11, 28, 42]. Also, nurses reported higher perceived stress levels [28, 30, 39, 42], psychopathological symptoms [15••], and higher PTSD symptoms, compared with other HCWs [15••, 29, 35, 40].

The level of exposure to infection seems to affect psychological outcomes. Studies showed that HCWs in units at high risk of infection present more severe mental health outcomes, compared with HCWs in units at low risk of infection [4, 6, $22,23,28,30,31 \bullet \bullet, 37,38,44]$. Interestingly, two studies found that being conscripted from a unit at low risk of infection to one at high risk of infection during an epidemic is a specific risk factor for worse mental health [6, 39]. Conversely, altruistically accepting the risk of infection is a protective factor [23, 44].

Similarly, direct contact with affected patients is a significant risk factor for all mental health outcomes $[8,12,15 \bullet \bullet, 18$, 27, 29, 39, 40].

With reference to more personal levels of exposure, studies show a trend for higher PTS and infection-related worries in HCWs who have been quarantined [3, 10, 44].

Two important organizational variables that emerge as protective factors for mental health outcomes are organizational support [10, 16, 30, 39] and perceived adequacy of training $[16,30,40]$.

Confidence in equipment and infection control measures appears to be a protective factor for mental health outcomes related to daily stress, namely, burnout [27, 30], perceived stress [9], and infection-related worries [33], but not for general mental health [29, 30, 37].

Moreover, disease-related risk perception was associated with worse mental health outcomes $[47,11,23,29,33,36$, 37, 42, 44].

\section{Discussion}

With this rapid review, we aimed to provide quantitative evidence on the potential maladaptive psychological outcomes in HCWs facing epidemic and pandemic situations and to identify potential risk and protective factors.

In describing the issues faced by HCWs responding to the COVID-19 pandemic, Kang et al. refers to "a high risk of infection and inadequate protection from contamination, overwork, frustration, discrimination, isolation, patients with negative emotions, a lack of contact with their families, and exhaustion" [47]. The evidence reviewed here, both related to the COVID-19 pandemic and to other previous epidemic/pandemic outbreaks, clearly confirms that facing such issues has a relevant psychological impact on $\mathrm{HCW}$ s responding to outbreaks.

In particular, during outbreaks, HCWs reported post-traumatic stress symptoms (11-73.4\%), depressive symptoms (27.5-50.7\%), insomnia (34-36.1\%), severe anxiety symptoms $(45 \%)$, general psychiatric symptoms (17.3-75.3\%), and high levels of work-related stress (18.1-80.1\%) [5, 6, 8, $11,12,15 \bullet \bullet, 24,25,33,38,39]$. Among these psychopathological outcomes, anxious and post-traumatic reactions were the most extensively investigated, and results pointed to the high prevalence of such areas of symptomatology in HCWs facing epidemic/pandemic outbreaks. This is not surprising, given the traumatic nature of the situations to which HCWs are exposed in their everyday work during epidemic/pandemic outbreaks. Furthermore, concerning mental health 


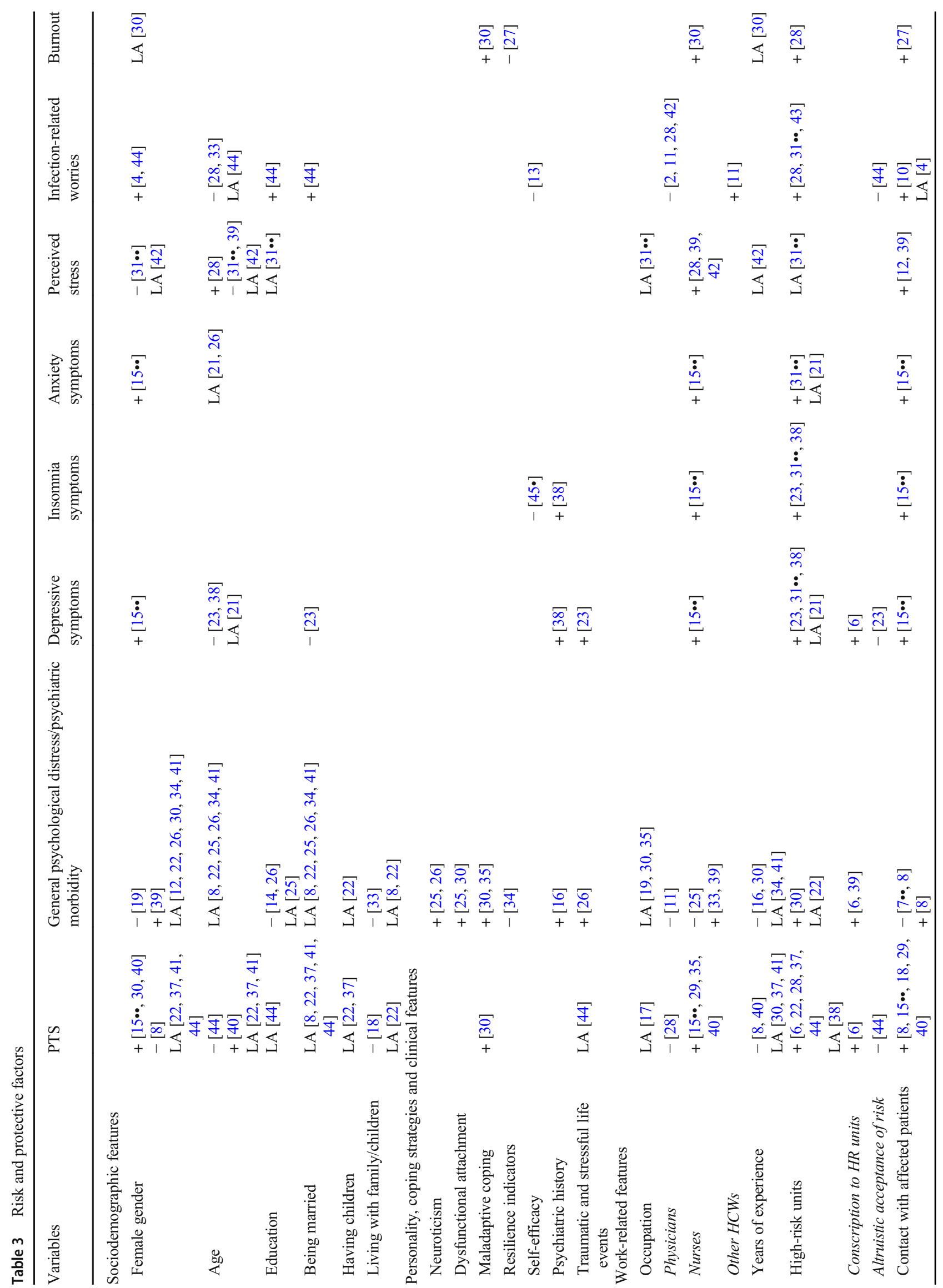




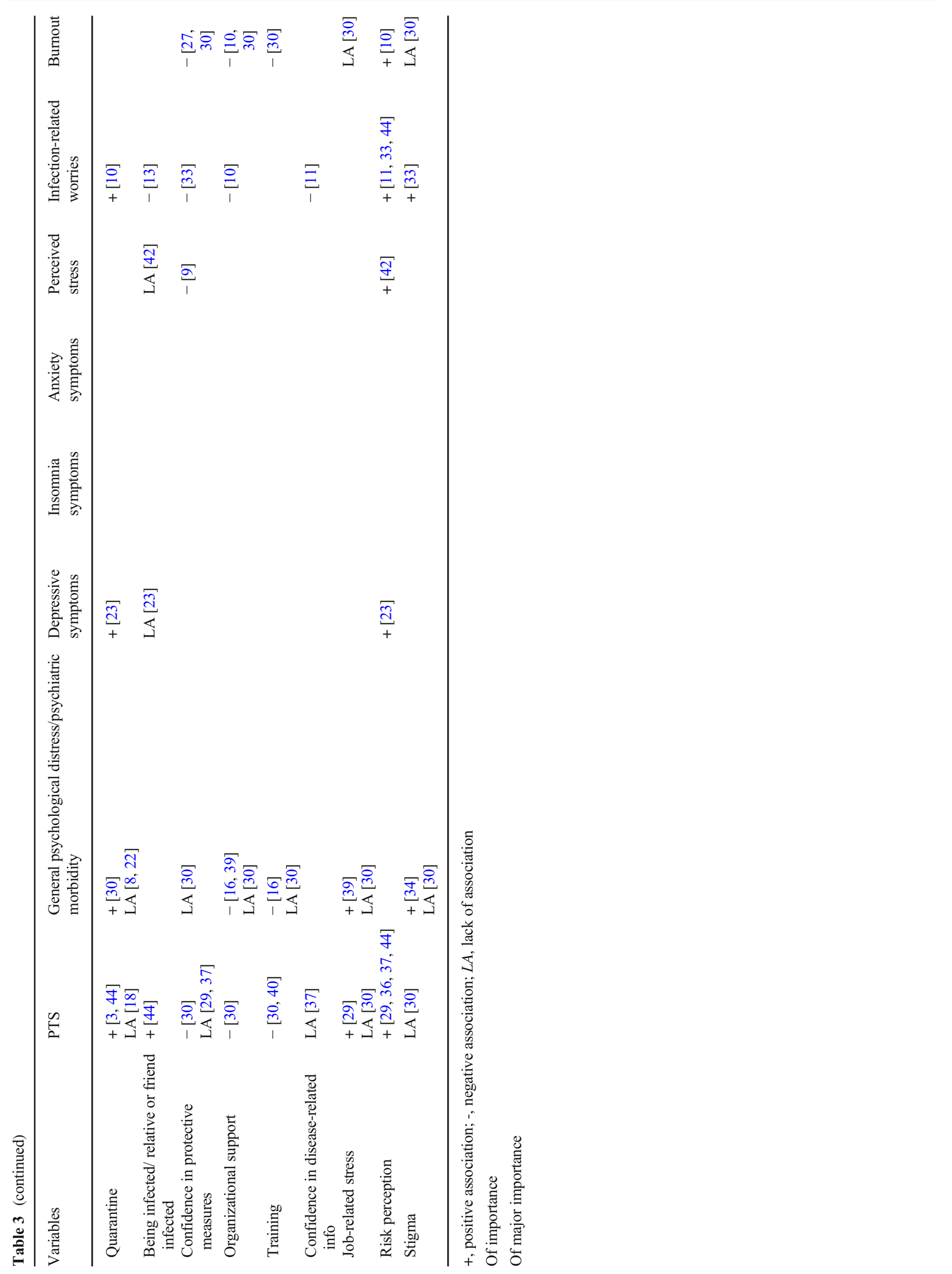


suffering, HCWs are considered a high risk group even in non-pandemic times [48].

Evidence related to psychopathological outcomes also shows that these maladaptive reactions can be long-lasting. In fact, post-traumatic and depressive symptoms, as well as general psychological distress, were reported even after periods ranging from 6 months up to 3 years after the epidemic/pandemic outbreak [23, 30, 35, 40, 41, 44].

At a psychological level, the evidence reviewed shows that general stress, specific infection-related worries, and workrelated stress are reported by HCWs facing epidemic/pandemic outbreaks. While stress and worries seem to be limited to the period of exposure to the outbreak, effects in terms of burnout can be long-lasting.

\section{What Can We Do to Reduce the Negative Impact of Outbreaks in Terms of Psychological Distress?}

During epidemic/pandemic outbreaks, HCWs are, of necessity, exposed to a situation that causes maladaptive psychological responses. However, in this review, we also provide evidence synthesis about personal and situational factors that showed to have an impact in determining the level of such maladaptive psychological responses.

In reviewing findings related to the role of sociodemographic factors, we did not find strong evidence suggesting that these personal factors make the difference in maladaptive psychological responses reported by HCWs. Instead, other personal factors are more consistently associated with poorer outcomes. HCWs with less effective coping abilities were more likely to report psychopathological responses, whereas those showing resilience were relatively less affected by the situation [27, 30, 34, 35]. Previous psychiatric history was also a predictor of higher maladaptive responses $[16,38]$.

A number of work-related features were associated with the level of maladaptive responses in HCWs. Results are particularly consistent in indicating that physicians are less psychologically affected than nurses in facing an epidemic/pandemic outbreak [2, 11, 28, 42]. This could be due to a higher physical contact with patients for nurses, as compared with physicians. Also, physicians could be more protected from these kinds of negative outcomes as a result of their longer training. Another situational factor that clearly emerges is the level of exposure to the epidemic/pandemic situation, with HCWs working in high-risk units (or being in contact with infected patients) reporting poorer psychological adjustment $[4,6,22,23,28$, $30,31 \bullet \bullet, 37,38,44]$. This is also consistent with results showing that higher risk perception is associated with higher maladaptive responses [10, 11, 23, 29, 33, 36, 37, 42, 44]. Considering work organization, confidence in protective measures, training, and organizational support were all related to less severe psychological outcomes $[9,10,16,27,30,33,39$, 40].

With these personal and situational factors in mind, we will try to provide some suggestions that can help reduce negative psychological responses of HCWs facing epidemic/pandemic outbreaks. These suggestions have the double aim of reducing the individual psychological burden of HCWs and strengthening the response capacity of healthcare systems.

\section{Know Your HCW Workforce to Support and Enhance Resilience and Coping Strategies}

Primary prevention should take place regularly, so that personal factors (e.g., past psychiatric history and difficulties in coping strategies) could be addressed. Such preventive interventions will result in a healthier workforce that will likely show better psychological responses in emergency situations, such as epidemic/pandemic outbreaks.

Training programs related to coping and resilience should be a regular part of HCWs' training and continuing education programs. Resilience trainings for HCWs have shown to be of benefit to health professionals (for a review, see Cleary and colleagues [49]). Training courses designed to build resilience to the stress of working during a pandemic are available also in online formats (e.g., Maunder and colleagues [50]). Furthermore, a recent review [51•] provided evidence that predisaster training and education can improve employees' confidence in their ability to cope with disasters. The need for training to enhance medical staff psychological skills was also recently underlined by Chen and colleagues in relation to the ongoing COVID-19 pandemic outbreak [52].

\section{Reserve a Special Attention to HCWs Working on the Frontline}

During the COVID-19 outbreak, frontline medical staff was included in the first priority category identified by the Chinese Society of Psychiatry to deliver psychological crisis intervention and provide technical guidance [53].

In China, on the one hand, psychological intervention teams, comprising psychological assistance hotline teams, and group activities to release stress were implemented for medical staff. On the other hand, the shift system and online platforms with medical advice were offered to help workers $[47,52]$. In a situation in which resources are limited, interventions should be focused, on a first stage, on frontline HCWs, since they are more likely to undergo maladaptive psychological consequences. Particular attention should be reserved to nurses, since results show that they are especially affected by a more intense physical exposure to infected patients. 


\section{Provide Adequate Protective Measures to HCWs}

The evidence synthesized in our review leads to hypothesize that when HCWs are provided with protective measures that are perceived as adequate, their risk perception is lower, and this could result in lower adverse psychological outcomes.

An essential factor to improve collaboration seems to be trust between organizations and workers [54, 55]. The feeling of being protected is associated with higher work motivation [56]. Hence, physical protective materials [54], together with frequent provision of information, should be provided.

\section{Organize Support Services That Can Be Delivered Online}

In comparison to previous epidemics, during COVID-19 pandemic, internet and smartphones are widely available [24]; hence, online mental health education, online psychological counseling services, and online psychological self-help intervention systems may and should be developed.

The major strength of the present rapid review is that, to the best of our knowledge, this is the first attempt to provide quantitative evidence of the mental health impact in HCWs facing epidemic and pandemic outbreaks as well as to identify potential related risk and protective factors.

Limitations are present as well. First, given the rapid nature of this literature review, we were not able to provide a quality of reporting evaluation of the included studies, which should represent the next step for a deeper understanding of the present results. However, we qualitatively evaluated the studies. Some limitations are related to the characteristics of the included studies: (1) the number of longitudinal studies was limited, and the majority were retrospective ones; (2) we found a great variability in the prevalence estimates, probably due to different cut-off scores used to identify cases (e.g., IESR) and to the use of heterogeneous instruments (e.g., stress perception); (3) it is possible that cultural differences in health beliefs, display of mental symptoms, and different healthcare systems have influenced the results of the studies included in this review; and (4) some risk and protective factors are still understudied in relation to psychological responses to epidemic/pandemic outbreaks; for example, perceived social support was investigated only by one recent study on COVID-19 [45•], despite evidence highlighting its protective role for mental health outcomes [46].

\section{Conclusions}

Our review confirms that HCWs responding to epidemic/pandemic outbreaks show a number of negative mental health and psychological consequences. Such sequelae are particularly alarming when considering their long-lasting nature and their plausible association with impaired decision-making capacities. As a matter of fact, we cannot avoid the exposure of HCWs to critical situations that could be detrimental for their mental health, since their rapid and effective deployment is critical in confronting epidemic/pandemic outbreaks. However, failing to consider the negative psychological impact that these workers suffer would result in consequences both at the individual level and in the healthcare response capacity at a systemic level.

We presented evidence that points to a number of personal and situational factors that play a role in mitigating or exacerbating the maladaptive consequences suffered by HCWs. Following empirical evidence, we proposed that assessment and promotion of coping strategies and resilience, special attention to frontline $\mathrm{HCWs}$, provision of adequate protective supplies, and organization of online support services could be ways to mitigate the negative psychological responses of HCWs responding to epidemic/pandemic outbreaks.

\section{Compliance with Ethical Standards}

Conflict of Interest The authors declare that they have no conflict of interest.

Human and Animal Rights and Informed Consent This article does not contain any studies with human or animal subjects performed by any of the authors.

\section{References}

Papers of particular interest, published recently, have been highlighted as:

- Of importance

- Of major importance

1. World Health Organization. Rapid reviews to strengthen health policy and systems: a practical guide. World Health Organization 2017. http://apps.who.int/iris/bitstream/10665/258698/1/ 9789241512763-eng.pdf

2. Alsubaie S, Hani Temsah M, Al-Eyadhy AA, Gossady I, Hasan GM, Al-Rabiaah A, et al. Middle East Respiratory Syndrome Coronavirus epidemic impact on healthcare workers' risk perceptions, work and personal lives. J Infect Dev Ctries. 2019;13(10): 920-6. https://doi.org/10.3855/jidc.11753.

3. Bai YM, Lin CC, Lin CY, Chen JY, Chue CM, Chou P. Survey of stress reactions among health care workers involved with the SARS outbreak. Psychiatr Serv. 2004;55(9):1055-7. https://doi.org/10. 1176/appi.ps.55.9.1055.

4. Bukhari EE, Temsah MH, Aleyadhy AA, Alrabiaa AA, Alhboob AA, Jamal AA, et al. Middle east respiratory syndrome coronavirus (MERS-CoV) outbreak perceptions of risk and stress evaluation in nurses. J Infect Dev Ctries. 2016;10(8):845-50. https://doi.org/10. 3855/jidc.6925.

5. Chan SSC, Leung GM, Tiwari AFY, Salili F, Leung SSK, Wong DCN, et al. The impact of work-related risk on nurses during the SARS outbreak in Hong Kong. Fam Community Heal. 2005;28(3): 274-87. https://doi.org/10.1097/00003727-200507000-00008. 
6. Chen CS, Wu HY, Yang P, Yen CF. Psychological distress of nurses in Taiwan who worked during the outbreak of SARS. Psychiatr Serv. 2005;56(1):76-9. https://doi.org/10.1176/appi.ps. 56.1.76.

7.• Chen NH, Wang PC, Hsieh MJ, Huang CC, Kao KC, Chen YH, et al. Impact of severe acute respiratory syndrome care on the general health status of healthcare workers in Taiwan. Infect Control Hosp Epidemiol. 2007;28(1):75-9. https://doi.org/10.1086/508824 This study is important for its longitudinal and case-control design.

8. Chong MY, Wang WC, Hsieh WC, Lee CY, Chiu NM, Yeh WC, et al. Psychological impact of severe acute respiratory syndrome on health workers in a tertiary hospital. Br J Psychiatry. 2004;185(1): 127-33. https://doi.org/10.1192/bjp.185.2.127.

9. Chua SE, Cheung V, Cheung C, McAlonan GM, Wong JWS, Cheung EPT, et al. Psychological effects of the SARS outbreak in Hong Kong on high-risk health care workers. Can J Psychiatry. 2004;49(6):391-3. https://doi.org/10.1177/070674370404900609.

10. Fiksenbaum L, Marjanovic Z, Greenglass ER, Coffey S. Emotional exhaustion and state anger in nurses who worked during the sars outbreak: the role of perceived threat and organizational support. Can J Community Ment Heal. 2006;25:89-103. https://doi.org/10. 7870/cjcmh-2006-0015.

11. Goulia P, Mantas C, Dimitroula D, Mantis D, Hyphantis T. General hospital staff worries, perceived sufficiency of information and associated psychological distress during the $\mathrm{A} / \mathrm{H} 1 \mathrm{~N} 1$ influenza pandemic. BMC Infect Dis. 2010;10:322. https://doi.org/10.1186/ 1471-2334-10-322.

12. Grace SL, Hershenfield K, Robertson E, Stewart DE. The occupational and psychosocial impact of SARS on academic physicians in three affected hospitals. Psychosomatics. 2005;46(5):385-91. https://doi.org/10.1176/appi.psy.46.5.385.

13. Ho SMY, Kwong-Lo RSY, Mak CWY, Wong JS. Fear of severe acute respiratory syndrome (SARS) among health care workers. J Consult Clin Psychol. 2005;73(2):344-9. https://doi.org/10.1037/ 0022-006X.73.2.344.

14. Ji D, Ji YJ, Duan XZ, Li WG, Sun ZQ, Song XA, et al. Prevalence of psychological symptoms among Ebola survivors and healthcare workers during the 2014-2015 Ebola outbreak in Sierra Leone: a cross-sectional study. Oncotarget. 2017;8(8):12784-91. https://doi. org/10.18632/oncotarget.14498.

15.• Lai J, Ma S, Wang Y, Cai Z, Hu J, Wei N, et al. Factors associated with mental health outcomes among health care workers exposed to coronavirus disease. JAMA. 2019;3(3):e203976. https://doi.org/10. $1001 /$ jamanetworkopen.2020.3976 This recent study is conducted on a representative sample of health-care workers involved in the COVID-19 outbreak.

16. Lancee WJ, Maunder RG, Goldbloom DS. Prevalence of psychiatric disorders among Toronto hospital workers one to two years after the SARS outbreak. Psychiatr Serv. 2008;59(1):91-5. https://doi. org/10.1176/ps.2008.59.1.91.

17. Lee AM, Wong JGWS, McAlonan GM, Cheung V, Cheung C, Sham PC, et al. Stress and psychological distress among SARS survivors 1 year after the outbreak. Can J Psychiatry. 2007;52: 233-40.

18. Lee SM, Kang WS, Cho AR, Kim T, Park JK. Psychological impact of the 2015 MERS outbreak on hospital workers and quarantined hemodialysis patients. Compr Psychiatry. 2018;87: 123-7. https://doi.org/10.1016/j.comppsych.2018.10.003.

19. Li L, Wan C, Ding R, Liu Y, Chen J, Wu Z, et al. Mental distress among Liberian medical staff working at the China Ebola Treatment Unit: a cross sectional study. Health Qual Life Outcomes. 2015;13:1-6. https://doi.org/10.1186/s12955-0150341-2.

20. Li Z, Ge J, Yang M, Feng J, Qiao M, Jiang R, et al. Vicarious traumatization in the general public, members, and non-members of medial teams aiding in COVID-19 control. Brain Behav Immun.
2020;S0889-1591(20):30309-3. https://doi.org/10.1016/j.bbi. 2020.03.007.

21. Liang Y, Chen M, Zheng X, Liu J. Screening for Chinese medical staff mental health by SDS and SAS during the outbreak of COVID-19. J Psychosom Res. 2020;133:16-8. https://doi.org/10. 1016/j.jpsychores.2020.110102.

22. Lin CY, Peng YC, Wu YH, Chang J, Chan CH, Yang DY. The psychological effect of severe acute respiratory syndrome on emergency department staff. Emerg Med J. 2007;24(1):12-7. https://doi. org/10.1136/emj.2006.035089.

23. Liu X, Kakade M, Fuller CJ, Fan B, Fang Y, Kong J, et al. Depression after exposure to stressful events: lessons learned from the severe acute respiratory syndrome epidemic. Compr Psychiatry. 2012;53(1):15-23. https://doi.org/10.1016/j.comppsych.2011.02. 003.

24. Liu S, Yang L, Zhang C, Xiang YT, Liu Z, Hu S, et al. Online mental health services in China during the COVID-19 outbreak. Lancet Psychiatry. 2020;7(4):e17-8. https://doi.org/10.1016/ S2215-0366(20)30077-8.

25. Lu YC, Shu BC, Chang YY, Lung FW. The mental health of hospital workers dealing with severe acute respiratory syndrome. Psychother Psychosom. 2006;75(6):370-5. https://doi.org/10. $1159 / 000095443$.

26. Lung FW, Lu YC, Chang YY, Shu BC. Mental symptoms in different health professionals during the SARS attack: a follow-up study. Psychiatr. 2009;80(2):107-16. https://doi.org/10.1007/ s11126-009-9095-5.

27. Marjanovic Z, Greenglass ER, Coffey S. The relevance of psychosocial variables and working conditions in predicting nurses' coping strategies during the SARS crisis: an online questionnaire survey. Int J Nurs Stud. 2007;44(6):991-8. https://doi.org/10.1016/j. ijnurstu.2006.02.012.

28. Matsuishi K, Kawazoe A, Imai H, Ito A, Mouri K, Kitamura N, et al. Psychological impact of the pandemic (H1N1) 2009 on general hospital workers in Kobe. Psychiatry Clin Neurosci. 2012;66(4): 353-60. https://doi.org/10.1111/j.1440-1819.2012.02336.x.

29. Maunder RG, Lancee WJ, Rourke S, Hunter JJ, Goldbloom D, Balderson K, et al. Factors associated with the psychological impact of severe acute respiratory syndrome on nurses and other hospital workers in Toronto. Psychosom Med. 2004;66(6):938-42. https:// doi.org/10.1097/01.psy.0000145673.84698.18.

30. Maunder RG, Lancee WJ, Balderson KE, Bennett JP, Borgundvaag B, Evans S, et al. Long-term psychological and occupational effects of providing hospital healthcare during SARS outbreak. Emerg Infect Dis. 2006;12(12):1924-32. https://doi.org/10.3201/eid1212. 060584.

31.• McAlonan GM, Lee AM, Cheung V, Cheung C, Tsang KWT, Sham PC, et al. Immediate and sustained psychological impact of an emerging infectious disease outbreak on health care workers. Can J Psychiatry, This study is important for its longitudinal and case-control design. 2007;52(4):241-7. https://doi.org/10. 1177/070674370705200406.

32. Mohammed A, Sheikh TL, Gidado S, Poggensee G, Nguku P, Olayinka A, et al. An evaluation of psychological distress and social support of survivors and contacts of Ebola virus disease infection and their relatives in Lagos, Nigeria: a cross sectional study 2014. BMC Public Health. 2015;15:1-8. https://doi.org/10.1186/ s12889-015-2167-6.

33. Nickell LA, Crighton EJ, Tracy CS, Al-Enazy H, Bolaji Y, Hanjrah $\mathrm{S}$, et al. Psychosocial effects of SARS on hospital staff: survey of a large tertiary care institution. CMAJ. 2004;170(5):793-8. https:// doi.org/10.1503/cmaj.1031077.

34. Park JS, Lee EH, Park NR, Choi YH. Mental health of nurses working at a government-designated hospital during a MERSCoV outbreak: a cross-sectional study. Arch Psychiatr Nurs. 2018;32(1):2-6. https://doi.org/10.1016/j.apnu.2017.09.006. 
35. Phua DH, Tang HK, Tham KY. Coping responses of emergency physicians and nurses to the 2003 severe acute respiratory syndrome outbreak. Acad Emerg Med. 2005;12(4):322-8. https://doi. org/10.1197/j.aem.2004.11.015.

36. Son H, Lee WJ, Kim HS, Lee KS, You M. Hospital workers' psychological resilience after the 2015 Middle East respiratory syndrome outbreak. Soc Behav Personal. 2019;47(2):1-13. https://doi. org $/ 10.3904 / \mathrm{kjim} .2018 .031$

37. Styra R, Hawryluck L, Robinson S, Kasapinovic S, Fones C, Gold WL. Impact on health care workers employed in high-risk areas during the Toronto SARS outbreak. J Psychosom Res. 2008;64(2):177-83. https://doi.org/10.1016/j.jpsychores.2007.07. 015.

38. Su TP, Lien TC, Yang CY, Su YL, Wang JH, Tsai SL, et al. Prevalence of psychiatric morbidity and psychological adaptation of the nurses in a structured SARS caring unit during outbreak: a prospective and periodic assessment study in Taiwan. J Psychiatr Res. 2007;41(1-2):119-30. https://doi.org/10.1016/j.jpsychires. 2005.12.006.

39. Tam CWC, Pang EPF, Lam LCW, Chiu HFK. Severe acute respiratory syndrome (SARS) in Hongkong in 2003: stress and psychological impact among frontline healthcare workers. Psychol Med. 2004;34(7):1197-204.https://doi.org/10.1017/ s0033291704002247.

40. Tang L, Pan L, Yuan L, Zha L. Prevalence and related factors of post-traumatic stress disorder among medical staff members exposed to H7N9 patients. Int J Nurs Sci. 2017;4(1):63-7. https:// doi.org/10.1016/j.ijnss.2016.12.002.

41. Tham KY, Tan YH, Loh OH, Tan WL, Ong MK, Tang HK. Psychological morbidity among emergency department doctors and nurses after the SARS outbreak. Hong Kong J Emerg Med. $2005 ; 12(5): 215-23$. https://doi.org/10.1177/ 102490790501200404 .

42. Wong TW, Yau JKY, Chan CLW, Kwong RSY, Ho SMY, Lau $\mathrm{CC}$, et al. The psychological impact of severe acute respiratory syndrome outbreak on healthcare workers in emergency departments and how they cope. Eur J Emerg Med. 2005;12:13-8. https://doi.org/10.1097/00063110-200502000-00005.

43. Wong SYS, Wong ELY, Chor J, Kung K, Chan PKS, Wong C, et al. Willingness to accept H1N1 pandemic influenza vaccine: a cross-sectional study of Hong Kong community nurses. BMC Infect Dis. 2010;10:107. https://doi.org/10.1186/1471-2334-10316.

44. Wu P, Fang Y, Guan Z, Fan B, Kong J, Yao Z, et al. The psychological impact of the SARS epidemic on hospital employees in China: exposure, risk perception, and altruistic acceptance of risk. Can J Psychiatry. 2009;54(5):302-11. https://doi.org/10.1177/ 070674370905400504.

45. Xiao H, Zhang Y, Kong D, Li S, Yang N. The effects of social support on sleep quality of medical staff treating patients with Coronavirus Disease 2019 (COVID-19) in January and February 2020 in China. Med Sci Monit. 2020;26:e923549. https://doi.org/ 10.12659/MSM.923549 This is the first study to investigate the impact of social support on the psychological well-being of health-care providers working during a pandemic crisis.
46. Tsai AC, Lucas M, Kawachi I. Association between social integration and suicide among women in the United States. JAMA Psychiatry. 2015;72(10):987-93. https://doi.org/10.1001/ jamapsychiatry.2015.1002.

47. Kang L, Li Y, Hu S, Chen M, Yang C, Yang BX, et al. The mental health of medical workers in Wuhan, China dealing with the 2019 novel coronavirus. Lancet Psychiatry. 2020;7(3):e14. https://doi. org/10.1016/S2215-0366(20)30047-X.

48. Dutheil F, Aubert C, Pereira B, Dambrun M, Moustafa F, Mermillod M, et al. Suicide among physicians and health-care workers: A systematic review and meta-analysis. PLoS One. 2019;14(12):1-28. https://doi.org/10.1371/journal.pone.0226361.

49. Cleary M, Kornhaber R, Thapa DK, West S, Visentin D. The effectiveness of interventions to improve resilience among health professionals: a systematic review. Nurse Educ Today. 2018;71: 247-63. https://doi.org/10.1016/j.nedt.2018.10.002.

50. Maunder RG, Lancee WJ, Mae R, Vincent L, Peladeau N, Beduz $\mathrm{MA}$, et al. Computer-assisted resilience training to prepare healthcare workers for pandemic influenza: a randomized trial of the optimal dose of training. BMC Health Serv Res. 2010;10:72. https://doi.org/10.1186/1472-6963-10-72.

51. Brooks SK, Dunn R, Amlôt R, Rubin GJ, Greenberg N. Social and occupational factors associated with psychological wellbeing among occupational groups affected by disaster: a systematic review. J Ment Health. 2017;26(4):373-84. https://doi.org/10.1080/ 09638237.2017 .1294732 This is a systematic review of social and occupational factors affecting the psychological wellbeing of health-care workers involed in the SARS outbreak.

52. Chen Q, Liang M, Li Y, Guo J, Fei D, Wang L, et al. Mental health care for medical staff in China during the COVID-19 outbreak. Lancet Psychiatry. 2020;7(4):e15-6. https://doi.org/10.1016/ S2215-0366(20)30078-X.

53. Jiang X, Deng L, Zhu Y, Ji H, Tao L, Liu L, et al. Psychological crisis intervention during the outbreak period of new coronavirus pneumonia from experience in Shanghai. Psychiatry Res. 2020;286:112903. https://doi.org/10.1016/j.psychres.2020. 112903.

54. Imai H. Trust is a key factor in the willingness of health professionals to work during the COVID-19 outbreak: experience from the H1N1 pandemic in Japan 2009. Psychiatry Clin Neurosci. 2020;74(5):329-30. https://doi.org/10.1111/pcn.12995.

55. Okello DRO, Gilson L. Exploring the influence of trust relationships on motivation in the health sector: a systematic review. Hum Resour Health. 2015;13:16. https://doi.org/10.1186/s12960-0150007-5.

56. Imai H, Matsuishi K, Ito A, Mouri K, Kitamura N, Akimoto K, et al. Factors associated with motivation and hesitation to work among health professionals during a public crisis: a cross sectional study of hospital workers in Japan during the pandemic (H1N1) 2009. BMC Public Health. 2010;10:672. https://doi.org/10.1186/1471-245810-672.

Publisher's Note Springer Nature remains neutral with regard to jurisdictional claims in published maps and institutional affiliations. 